\title{
Morphological Aspects of the Parietal Lobe in the Lateral Surface of the Brain: An in vivo MRI Study
}

\section{Sergio Georgeto MD*}

Universidade Estadual de Londrina Centro de Ciencias da Saude, Brazil

\begin{abstract}
Background: parietal lobe is an important area of the brain involved in a number of higher cortical functions. Anatomy descriptions differ with regard to the classical ways, making the location of the elements that compose the imprecise parietal lobule. Objective: To develop a method of location nine references in the parietal lobe, analyze the reliability of this method when using the techniques T1 and T1 IR GRE GRE and determine which technique performs better in identifying references proposals.

Methods: The study included 30 healthy volunteers with a mean age of $25.3 \pm 7.04$ years; $1653.3 \%$ were women and $1446.7 \%$ were men. Methodology sought to identify the structures that comprise the parietal lobe. The reliability of the method was evaluated on technical GRE T1 and T1 IR GRE in two stages. Firstly, intra-observer analysis and inter-observer analyzes were performed using individuals as a parameter. Then, the techniques were compared with respect to hemispheres, using techniques such as a parameter. The performance of each technique was evaluated using a binomial test considering two categories of display method comprising references.
\end{abstract}

Results: Both techniques have shown a satisfactory or higher level, both intra and inter-rater of most references. The T1 IR GRE technique achieved superior performance in easily classified as identifiable.

Conclusions: The proposed method was reliable in identifying references located in the parietal lobe. T1 IR GRE was the technique that outperformed.

Keywords: Magnetic resonance imaging; MRI; Parietal lobe anatomy; Anatomical structures of the brain

\section{Introduction}

Understanding the anatomy of the parietal lobe PL is important because of the multiple functions performed by this region and its status as the location where various pathologies occur. Mesulan observed that the shape of the elements comprising the cortical mantle is related to the pattern of subcortical connections, notably relating the morphology of the cortex to the function performed by this group of neurons [1]. As a result, determination of the main elements comprising a given region may translate into the way this region carries out its functions. The classic descriptions in the literature by Ono et al. based on anatomical studies, and Duvernoy, which correlated anatomical features and MRI with the main references comprising the PL, have been questioned for presenting inconsistent and diverging assertions $[2,3]$. In this context, a new study on this region of the brain, with a reassessment of previous findings and confirmation of current findings, is deemed important. Throughout the years, findings from neuroanatomical studies in formaldehyde-fixed brains have been extrapolated to establish in vivo correlations. Anatomical studies of normal individuals using MRI are now possible, making it easier to understand the singularities and similarities of each region. There is no standard method for studying topographical morphology through MRI, and no one sequence is used routinely. Recently, the following methods have been applied: T1weighted gradient echo T1 GRE [4], T2-weighted gradient echo T2 GRE [5], spoiled GRASS [6], spoiled GRE [7], and T1 IR GRE [8,9]. Given the improvements in MRI equipment, techniques such as the T1-weighted inversion recovery gradient echo T1 IR GRE are no longer economically unfeasible and may be used routinely. It is important to highlight that this technique features a higher contrast between the cortical grey matter and the subcortical white matter, making the borders of the gyrus clearer and potentially easier to delineate [10]. Geurts et al. demonstrated the utility of this technique in assessing cortical lesions resulting from multiple sclerosis [9], while Achten et al. highlighted its importance in visualising cryptogenic neocortical lesions and atrophy of the hippocampus associated with epilepsy in the temporal lobe [11]. There is an apparent difficulty in anatomical description of the lateral surface of the brain in relation to the location of the sulci and gyri which comprise the encephalic surface. This results from the difficulty in assessing the surface through imaging exams, and from the individual variability of the cortical topography. Without reference points, identifying the most relevant structures becomes difficult and confusing. Consequently, methodology adapted from descriptions by Naidich et al. and Wagner et al. was further adapted into a nine-step method for identifying nine references which comprise the parietal lobe on the lateral surface $[12,13]$. In this context, the objective of this is study is to propose a method to locate nine references in the parietal lobe, analyse the reliability of the techniques T1 GRE and T1 IR GRE in relation to the method, determine which technique performs better in identifying the proposed references, and discuss controversial points found in the literature regarding the topographical descriptions of this region.

\section{Subjects and Methods}

\section{Sample size and participants}

For the sample selection, a statistical power of $80 \%$ was considered.

*Corresponding author: Sergio Georgeto MD, Universidade Estadual de Londrina Centro de Ciencias da Saude, Brazil, Tel +55 43 991110088; E-mail: georgetosm@gmail.com

Received April 06, 2017; Accepted April 10, 2017; Published April 15, 2017

Citation: Sergio Georgeto MD (2017) Morphological Aspects of the Parietal Lobe in the Lateral Surface of the Brain: An in vivo MRI Study. Med Rep Case Stud 2 130. doi: $10.4172 / 2572-5130.1000130$

Copyright: ( 2017 Sergio Georgeto MD. This is an open-access article distributed under the terms of the Creative Commons Attribution License, which permits unrestricted use, distribution, and reproduction in any medium, provided the original author and source are credited. 
A moderate kappa kappa $>0.5$ was used to test the null hypothesis that there is no agreement between the methods for identifying the structures on the lateral surface of the brain. From those parameters, according to Sim and Wright, the minimum sample was calculated as 25 individuals was stated by Sim and Wright [14]. Considering potential losses, 30 individuals were selected. The study included 30 adult volunteers selected in a non-probabilistic fashion with a mean age of $25.3 \pm 7.04$ years; $1653.3 \%$ were women and $1446.7 \%$ were men. After approval by the ethics committee, the volunteers were interviewed so that the eligibility criteria could be verified and the procedures could be explained to them. They were then asked to sign an informed consent form.

\section{Image acquisition processes}

All 30 individuals underwent a single standard cranial MRI, and images were acquired using the T1 GRE and T1 IR GRE pulse sequences. The MRI equipment was a Magneton Symphony $1.5 \mathrm{~T}$ SIEMENS, Erlangen, Germany, with a 12-channel coil. The isotropic volumetric images were obtained using two sequences, T1 GRE and T1 IR GRE, following the standard specifications listed below and on planes parallel to the inter commissural line. The T1 GRE images were acquired with a $15^{\circ}$ flip angle. For the inversion recovery imaging T1 IR GRE, inversion time was 350 milliseconds; the parameters for each sequence are detailed in Table 1.

The images obtained were initially assessed by an experienced radiologist in order to eliminate images with movement artefacts, images which were not adequate for the assessment, and images containing pathological findings the quality of the images acquired using T1 GRE and T1 IR GRE on the sagittal plane which were used in this study. The resulting data were recorded in the DICOM Digital Imaging and Communications in Medicine system, where the images were codified to secure volunteer anonymity. The images were transferred to a MacBook Pro 15" notebook, and Osiri X M.D. ${ }^{\circ}$ v.5.7.1. 64 bit software Pixmeo SARL, Bernex, Switzerland was used to analyse the images through 2D orthogonal MPR multi-planar reconstruction. The T1 GRE and T1 IR GRE files formed 60 blocks of images, and the left and right hemispheres were analysed separately, resulting in a total of 120 blocks. Five assessment sequences were produced at random to conduct the repeatability and reproducibility tests, and the AES Advanced Encryption Standard method was used. The advantage of this method is the use of a single key to encrypt and decrypt the code. The software performed in a completely satisfactory manner considering the project requirements. It was developed by Alekcey Colione and is available at Ale.inf.br/encoder.

\section{Method to Identify the Structures in the Parietal Lobe}

The anatomy of the lateral surface of the brain was assessed in a qualitative manner with regard to identifying the most important anatomical structures. Methodology adapted from a study by Naidich et al. [12] and Wagner et al. [13] was used, following nine steps to determine the nine references for the parietal lobe, as follows:

\section{Stage 1}

Obtain a sagittal image of the convexity: On the sagittal plane, parallel to the midline, the image with the lateral fissure LF in its largest extension is chosen. The image may later be repositioned laterally or medially in order to identify the following references.

\section{Stage 2}

Postcentral sulcus PoCS: Immediately posterior to the postcentral gyrus, this structure is oblique and parallel to this gyrus. The upper portion of the postcentral sulcus may extend horizontally, continuing along the intraparietal sulcus.

\section{Stage 3}

Supramarginal gyrus SMG: The reference point for the supramarginal gyrus is the posterior ascending ramus of the lateral fissure and the inferior portion of the postcentral sulcus. The posterior ascending ramus is located in the superior portion and its distal edge features a continuous, bifid, or hook-like shape. Above this landmark is a band of tissue which begins at the inferior portion of the postcentral sulcus and includes the posterior portion, forming a C-shape. It then joins the superior temporal gyrus. The extension of this structure was determined according to the protrusion of the parietal operculum.

\section{Stage 4}

Superior temporal sulcus STS: Parallel to the lateral fissure and identified as a long, continuous, and horizontal sulcus that may feature a simple or bifid edge in its supradistal portion.

\section{Stage 5}

Angular gyrus AG: Identified as the brain tissue around the distal and angular portion of the superior temporal sulcus. The angular gyrus may be U-shaped, with a simple edge, or heart-shaped, in which case it has a bifid edge.

\section{Stage 6}

Intraparietal sulcus IPS: The intraparietal sulcus may be observed as a primary sulcus with an inferior concavity, emerging from the postcentral sulcus and forming an arch above the postcentral gyrus, the angular gyrus, and any accessory gyrus that extends toward the occipital lobe. It may also be recognised as a discontinuous sulcus with parts located behind the supramarginal sulcus, a secondary sulcus which is posterior to the angular gyrus, or an intermediary sulcus

\begin{tabular}{|c|c|c|c|c|}
\hline & \multicolumn{2}{|c|}{ T1 GRE } & \multicolumn{2}{|c|}{ T1 IR GRE } \\
\hline Acquisition & \multicolumn{2}{|c|}{ Gradient echo sagittal plane } & \multicolumn{2}{|c|}{ Coronal plane } \\
\hline Matrix & \multicolumn{2}{|c|}{$256 \times 256$} & \multicolumn{2}{|c|}{$256 \times 256$} \\
\hline Voxel & \multicolumn{2}{|c|}{ Isotropic $(1 \times 1 \times 1 \mathrm{~mm})$} & \multicolumn{2}{|c|}{ Isotropic $(1 \times 1 \times 1 \mathrm{~mm})$} \\
\hline \multirow{2}{*}{ Parameters } & TR & 1910 & TR & 4000 \\
\hline & $\mathrm{TE}$ & 3.09 & TE & 373 \\
\hline FOV & \multicolumn{2}{|c|}{$256 \mathrm{~mm}$} & \multicolumn{2}{|c|}{$260 \mathrm{~mm}$} \\
\hline Slice thickness & \multicolumn{2}{|c|}{$1 \mathrm{~mm}$} & \multicolumn{2}{|c|}{$1 \mathrm{~mm}$} \\
\hline Spacing between slices & \multicolumn{2}{|c|}{ Zero } & \multicolumn{2}{|c|}{ Zero } \\
\hline Flip angle & \multicolumn{2}{|c|}{$15^{\circ}$} & \multicolumn{2}{|c|}{-} \\
\hline Inversion time & \multicolumn{2}{|c|}{-} & \multicolumn{2}{|c|}{$350 \mathrm{msec}$} \\
\hline
\end{tabular}

Table 1: Specification of the MRI acquisition parameters for both sequences (T1GRE and T1 IR GRE). 
positioned dorsally in relation to a pre-supramarginal or pre-angular accessory sulcus. A third description for the intraparietal sulcus is the sulcus that defines the supramarginal gyrus, the angular gyrus, and the small temporal-occipital arch which together form the inferior parietal lobe.

\section{Stage 7}

Superior parietal lobe SPL: Group of gyri which are in a posterior position in relation to the superior portion of the postcentral gyrus, and a superior position in relation to the supramarginal and angular gyri. Its limits are established by the superior portion of the postcentral sulcus on the anterior side and by the intraparietal sulcus on the inferior side.

\section{Stage 8}

The U sign US: Inferior junction between the precentral gyrus and the postcentral gyrus, located under point where the central sulcus ends; it is shaped like an arch with the concavity facing upwards. The postcentral gyrus is located immediately posterior to this structure.

\section{Stage 9}

Thickness, PostCG < PreCG TPoCG $<$ PreCG: Apparent narrowing in the postcentral gyrus PostCG compared to the precentral gyrus PreCG when observed along the central sulcus in sagittal imaging.

\section{Data Analysis}

The reliability of this method of data analysis was tested based on assessments of repeatability intra-observer analyses and reproducibility inter-observer analyses, as well as on the level of agreement between the techniques for each of the proposed references in relation to each hemisphere. Next, the performance between the two sequences was compared using the significant difference between the proportions of easily identified findings from each technique. In the intra-rater and inter-rater analyses, both techniques were assessed in relation to the references in each hemisphere. The agreement level was determined through the kappa method for each technique in relation to the side. An overall kappa was calculated for all references according to the technique and in relation to the side studied. In order to verify the level of agreement and compare the techniques in each hemisphere, the items comprising the parietal region in each MRI were classified as: easily identified, unclear, and not identified. In other words, it was possible to verify the agreement between the techniques and between hemispheres using the kappa. To perform this comparison, assessments deemed unclear were merged with those where the structures were not identified, resulting in only two possible assessments with regard to the identification of the item in each MRI. This permitted the use of the binomial method to compare the identification proportions between the techniques in relation to the hemisphere. For evaluation of the parietal region as a whole, all nine references were grouped in such a way that the number of evaluations in the group represented the total number of items multiplied by the number of patients evaluated. Data were assessed using Stata v.13.1. software StataCorpor. Stata Statistical Software: Release [13]. College Station, TX: Stat. Stata Corp LP. For the repeatability analysis, the researcher performed three assessments with an interval of ten days between each observation. As for the reproducibility of the method, analyses were performed by comparing the researcher's first assessment with two external assessments conducted by two neurosurgeons who were invited to participate for being familiar with both the anatomy in the region and the corresponding imaging. They received an instruction manual with relevant information on the variables to be observed and an explanation of the use of the method, following the same protocol used in the assessment of repeatability. In order to classify the agreement pattern in both the repeatability and reproducibility assessments, a prevalenceadjusted and bias-adjusted kappa PABAK was used, as proposed by Byrt et al. Kappa values equal to or greater than satisfactory were deemed good agreement values, as detailed in Table 2 [15].

\section{Results}

A satisfactory or higher level of agreement was found for the two techniques in both the intra- and inter-rater analyses of most references. Reports obtained through the different techniques in each hemisphere can be found in Table 3. The superior temporal sulcus stands out because of the lack of disagreement on the right side using the T1 IR GRE technique. On the left side, however, disagreement was more apparent. Regarding the T1 GRE technique, disagreement was more apparent on both sides, indicating that, both in the intra- and inter-observer analyses, it was more difficult to find agreements in the assessments using T1 GRE than using T1 IR GRE.

Continuing with the reliability test, the method was checked for the level of agreement between the techniques for each proposed reference in relation to each hemisphere. A satisfactory or higher level of agreement was observed between the two techniques in the parietal region as a whole on both the left and right sides. Table 4 shows that the superior temporal sulcus and the superior parietal lobule featured a weak level of agreement on the left side. The percentage of disagreement, in turn, was high for both items and on both the right and left sides. This indicates that several assessments were perceived differently in the different techniques. For most items, however, both techniques showed a satisfactory or higher level of agreement regarding the ease of identifying the references. No disagreements were observed with regard to the assessments. Analysis using the binomial test was applied to assess the performance between T1 GRE and T1 IR GRE. The assessment considered the significant difference between the proportions of easily identified findings in each technique. Table 5 showed a significant difference in the parietal region as a whole both on the right and left sides. The T1 IR GRE technique was superior on both sides. The superior temporal sulcus STS and the angular gyrus AG stood out. By assessing the references individually using T1 IR GRE, the STS was significantly easy to identify on both sides, while the angular gyrus was only significantly easy to identify on the right. The intraparietal sulcus and the postcentral sulcus were easily identified by both techniques in all assessments. A bar graph was created to provide a better visualisation of the percentages of easily-identified findings. It lists references individually and shows all nine items in relation to the technique used and side studied.

\section{Discussion}

For the objectives of the study to be achieved, it was necessary to first assess the reliability of the method for the selected techniques. This was done by analysing intra- and inter-observer agreement and

\begin{tabular}{|c|c|}
\hline $0.93-1.00$ & Excellent \\
\hline $0.81-0.92$ & Very good \\
\hline $0.61-0.80$ & Good \\
\hline $0.41-0.60$ & Satisfactory \\
\hline $0.21-0.40$ & Weak \\
\hline $0.01-0.20$ & Poor \\
\hline 0.00 or less & No agreement \\
\hline
\end{tabular}

Table 2: Pattern of agreement with prevalence-adjusted and bias-adjusted kappa according to criteria by Byrt et al. [15]. 
Page 4 of 7

\begin{tabular}{|c|c|c|c|c|c|c|}
\hline \multirow[t]{2}{*}{ Variable } & \multicolumn{3}{|c|}{ Intra-observer } & \multicolumn{3}{|c|}{ Inter-observer } \\
\hline & Disagrees (\%) & KAPPA & $P$ value & Disagrees (\%) & KAPPA & $P$ value \\
\hline \multicolumn{7}{|l|}{ T1 IR GRE - Right Side } \\
\hline References for the parietal region & $16(5.9)$ & 0.41 & $<0.001$ & $13(4.8)$ & 0.6 & $<0.001$ \\
\hline \multirow[t]{2}{*}{ Postcentral gyrus } & $0(0)$ & - & - & $0(0)$ & - & - \\
\hline & $0(0)$ & - & - & $0(0)$ & - & - \\
\hline \multirow[t]{2}{*}{ Posterior ascending ramus/SMG } & $0(0)$ & - & - & $1(3.3)$ & - & - \\
\hline & $1(3.3)$ & - & - & $3(10)$ & 0.36 & $<0.001$ \\
\hline Angular gyrus & $8(26.7)$ & 0.47 & $<0.001$ & $4(13.3)$ & 0.78 & $<0.001$ \\
\hline Intraparietal sulcus & $1(3.3)$ & - & - & $1(3.3)$ & 0.49 & $<0.001$ \\
\hline Superior parietal lobe & $2(6.7)$ & - & - & $0(0)$ & 1 & $<0.001$ \\
\hline U-shaped connection & $4(13.3)$ & - & - & $4(13.3)$ & - & - \\
\hline Thickness, PostCG<PreCG & $0(0)$ & - & - & $0(0)$ & - & - \\
\hline \multicolumn{7}{|l|}{ T1 IR GRE - Left side } \\
\hline References for the parietal region & $30(11.1)$ & 0.63 & $<0.001$ & $29(10.7)$ & 0.69 & $<0.001$ \\
\hline Postcentral gyrus & $0(0)$ & - & - & $0(0)$ & - & - \\
\hline Postcentral sulcus & $0(0)$ & - & - & $0(0)$ & - & - \\
\hline Posterior ascending ramus/SMG & $0(0)$ & 1 & $<0.001$ & $1(3.3)$ & 0.94 & $<0.001$ \\
\hline Superior temporal sulcus & $9(30)$ & $0.24^{\star * *}$ & 0.012 & $8(26.7)$ & 0.55 & $<0.001$ \\
\hline Angular gyrus & $15(50)$ & $0.33^{* * *}$ & 0.001 & $9(30)$ & 0.59 & $<0.001$ \\
\hline Intraparietal sulcus & $0(0)$ & - & - & $0(0)$ & - & - \\
\hline Superior parietal lobe & $2(6.7)$ & 0.58 & $<0.001$ & $3(10)$ & $0.22^{\star \star \star}$ & 0.021 \\
\hline U-shaped connection & $4(13.3)$ & $0.15^{\star * *}$ & 0.0734 & $8(26.7)$ & - & - \\
\hline Thickness, PostCG<PreCG & $0(0)$ & - & - & $0(0)$ & - & - \\
\hline \multicolumn{7}{|l|}{ T1 GRE - Right Side } \\
\hline References for the parietal region & $35(13)$ & 0.47 & $<0.001$ & $31(11.5)$ & 0.73 & $<0.001$ \\
\hline Postcentral gyrus & $0(0)$ & - & - & $0(0)$ & - & - \\
\hline Postcentral sulcus & $0(0)$ & - & - & $0(0)$ & - & - \\
\hline Posterior ascending ramus/SMG & $2(6.7)$ & - & - & $3(10)$ & $0.36^{* \star *}$ & $<0.001$ \\
\hline Superior temporal sulcus & $12(40)$ & $0.17^{* *}$ & 0.057 & $5(16.7)$ & 0.64 & $<0.001$ \\
\hline Angular gyrus & $13(43.3)$ & 0.42 & $<0.001$ & $4(13.3)$ & 0.82 & $<0.001$ \\
\hline Intraparietal sulcus & $0(0)$ & - & - & $0(0)$ & - & - \\
\hline Superior parietal lobe & $4(13.3)$ & $0.15^{\star *}$ & 0.073 & $1(3.3)$ & 0.84 & $<0.001$ \\
\hline U-shaped connection & $4(13.3)$ & - & - & $7(23.3)$ & - & - \\
\hline Thickness, PostCG<PreCG & $0(0)$ & - & - & $0(0)$ & - & - \\
\hline \multicolumn{7}{|l|}{ T1 GRE - Left Side } \\
\hline References for the parietal region & $30(11.1)$ & 0.73 & $<0.001$ & $30(11.1)$ & 0.73 & $<0.001$ \\
\hline Postcentral gyrus & $0(0)$ & - & - & $0(0)$ & - & - \\
\hline Postcentral sulcus & $0(0)$ & - & - & $0(0)$ & - & - \\
\hline Posterior ascending ramus/SMG & $0(0)$ & 1 & $<0.001$ & $1(3.3)$ & 0.94 & $<0.001$ \\
\hline Superior temporal sulcus & $15(50)$ & $0.32^{* * *}$ & 0.001 & $13(43.3)$ & $0.39^{* * *}$ & $<0.001$ \\
\hline Angular gyrus & $9(30)$ & 0.54 & $<0.001$ & $8(26.7)$ & 0.56 & $<0.001$ \\
\hline Intraparietal sulcus & $0(0)$ & - & - & $0(0)$ & - & - \\
\hline Superior parietal lobe & $2(6.7)$ & 0.64 & $<0.001$ & $2(6.7)$ & 0.58 & $<0.001$ \\
\hline U-shaped connection & $4(13.3)$ & $0.15^{\star * \star}$ & 0.0734 & $4(13.3)$ & $0.15^{\star \star \star}$ & 0.0734 \\
\hline Thickness, PostCG<PreCG & $0(0)$ & - & - & $0(0)$ & - & - \\
\hline
\end{tabular}

$\mathrm{No}^{*}$, poor* , and weak*** agreement.

Table 3: Agreement between techniques in intra- and inter-observer assessments in relation to the hemisphere.

comparing the techniques in relation to the references in each hemisphere. The reliability of the method to analyse repeatability intraobserver and reproducibility inter-observer used the individuals as a parameter. It showed a satisfactory or higher level of agreement using the two techniques in both hemispheres and in the intra-rater and inter-rater observations. The reliability of the method to compare the techniques in relation to the hemispheres used the techniques as a parameter. It showed a satisfactory or higher level of agreement for both the right and left parietal regions, and yielded a total kappa of 0.53 on the right and 0.75 on the left. By isolating the references, the elements that featured the highest percentage of easily identified findings were the $\mathrm{U}$-shaped connection, postcentral gyrus, and postcentral sulcus, regardless of the technique used and side studied.
These findings are compatible with those described by Wagner et al. [13]. The exceptions found in this study were related to the superior temporal sulcus STS on both sides, the angular gyrus AG on the right side, and the superior parietal lobule SPL on the left. In order to clarify possible causes that led to these results, the findings were assessed individually. For the STS, the T1 IR GRE method proved to be better than the T1 GRE with respect to identification on both sides. Data from an anatomical study helped interpret how the STS is more likely to appear according to the hemisphere [16]. The higher the number of segmentations, the harder it was to define the sulcus correctly in MRI assessment. By observing formaldehyde-fixed brains, Ono et al. found that the STS was continuous or had at least two interruptions on the right in $84 \%$ of hemispheres and on the left in $60 \%$ of hemispheres. The 
Citation: Sergio Georgeto MD (2017) Morphological Aspects of the Parietal Lobe in the Lateral Surface of the Brain: An in vivo MRI Study. Med Rep Case Stud 2: 130. doi: 10.4172/2572-5130.1000130

Page 5 of 7

\begin{tabular}{|c|c|c|c|c|c|c|}
\hline Variable & $\begin{array}{l}\text { Agrees (not } \\
\text { identified) }\end{array}$ & Agrees (Unclear) & $\begin{array}{l}\text { Agrees (easily } \\
\text { identified) }\end{array}$ & Disagrees & KAPPA & $P$ value \\
\hline \multicolumn{7}{|l|}{ T1 GRE - Right Side } \\
\hline $\begin{array}{l}\text { References for the parietal } \\
\text { region }\end{array}$ & $0(0)$ & $11(4.1)$ & $242(89.6)$ & $17(6.3)$ & 0.53 & $<0.001$ \\
\hline Postcentral gyrus & $0(0)$ & $0(0)$ & $30(100)$ & $0(0)$ & - & - \\
\hline Postcentral sulcus & $0(0)$ & $0(0)$ & $30(100)$ & $0(0)$ & - & - \\
\hline $\begin{array}{l}\text { Posterior ascending ramus/ } \\
\text { SMG }\end{array}$ & $0(0)$ & $0(0)$ & $29(96.7)$ & $1(3.3)$ & 0.65 & $<0.001$ \\
\hline Superior temporal sulcus & $0(0)$ & $1(3.3)$ & $21(70)$ & $8(26.7)$ & $0.15^{* *}$ & 0.060 \\
\hline Angular gyrus & $0(0)$ & $7(23.3)$ & $17(56.7)$ & $6(20)$ & 0.57 & $<0.001$ \\
\hline Intraparietal sulcus & $0(0)$ & $0(0)$ & $30(100)$ & $0(0)$ & - & - \\
\hline Superior parietal lobe & $0(0)$ & $1(3.3)$ & $27(90)$ & $2(6.7)$ & 0.47 & 0.001 \\
\hline U-shaped connection & $0(0)$ & $2(6.7)$ & $28(93.3)$ & $0(0)$ & 1 & $<0.001$ \\
\hline Thickness, PostCG<PreCG & $0(0)$ & $0(0)$ & $30(100)$ & $0(0)$ & - & - \\
\hline \multicolumn{7}{|l|}{ T1 GRE - Left Side } \\
\hline $\begin{array}{l}\text { References for the parietal } \\
\text { region }\end{array}$ & $0(0)$ & $33(12.2)$ & $219(81.1)$ & $18(6.7)$ & 0.75 & $<0.001$ \\
\hline Postcentral gyrus & $0(0)$ & $0(0)$ & $30(100)$ & $0(0)$ & - & - \\
\hline Postcentral sulcus & $0(0)$ & $0(0)$ & $30(100)$ & $0(0)$ & - & - \\
\hline $\begin{array}{l}\text { Posterior ascending ramus/ } \\
\text { SMG }\end{array}$ & $0(0)$ & $8(26.7)$ & $22(73.3)$ & $0(0)$ & 1 & $<0.001$ \\
\hline Superior temporal sulcus & $0(0)$ & $7(23.3)$ & $13(43.3)$ & $10(33.3)$ & $0.35^{\star \star *}$ & 0.012 \\
\hline Angular gyrus & $0(0)$ & $14(46.7)$ & $11(36.7)$ & $5(16.7)$ & 0.67 & $<0.001$ \\
\hline Intraparietal sulcus & $0(0)$ & $0(0)$ & $30(100)$ & $0(0)$ & - & - \\
\hline Superior parietal lobe & $0(0)$ & $1(3.3)$ & $26(86.7)$ & $3(10)$ & $0.35^{\star * *}$ & 0.026 \\
\hline U-shaped connection & $0(0)$ & $3(10)$ & $27(90)$ & $0(0)$ & 1 & $<0.001$ \\
\hline Thickness, PostCG<PreCG & $0(0)$ & $0(0)$ & $30(100)$ & $0(0)$ & - & - \\
\hline
\end{tabular}

$\mathrm{No}^{*}$, poor* $^{* *}$, and weak ${ }^{* * *}$ agreement

Table 4: Agreement between the T1 GRE and T1 IR GRE techniques in relation to the references in each hemisphere.

\begin{tabular}{|c|c|c|c|c|c|}
\hline & \multicolumn{2}{|c|}{ IR - Easily Identified } & \multicolumn{2}{|c|}{ T1 - Easily Identified } & \multirow{2}{*}{$\begin{array}{c}\text { T1 vs. IR } \\
\text { P value }\end{array}$} \\
\hline & No & Yes & No & Yes & \\
\hline \multicolumn{6}{|l|}{ Right Side } \\
\hline References for the parietal region & $11(4.1 \%)$ & $259(5.9 \%)$ & $28(10.4 \%)$ & $242(89.6 \%)$ & 0.002 \\
\hline Postcentral gyrus & $0(0 \%)$ & $30(100 \%)$ & $0(0 \%)$ & $30(100 \%)$ & --- \\
\hline Postcentral sulcus & $0(0 \%)$ & $30(100 \%)$ & $0(0 \%)$ & $30(100 \%)$ & --- \\
\hline Posterior ascending ramus/SMG & $0(0 \%)$ & $30(100 \%)$ & $1(3.3 \%)$ & $29(96.7 \%)$ & 1 \\
\hline Superior temporal sulcus & $1(3.3 \%)$ & $29(96.7 \%)$ & $9(30 \%)$ & $21(70 \%)$ & 0.008 \\
\hline Angular gyrus & $7(23.3 \%)$ & $23(76.7 \%)$ & $13(43.3 \%)$ & $17(56.7 \%)$ & 0.031 \\
\hline Intraparietal sulcus & $0(0 \%)$ & $30(100 \%)$ & $0(0 \%)$ & $30(100 \%)$ & --- \\
\hline Superior parietal lobe & $1(3.3 \%)$ & $29(96.7 \%)$ & $3(10 \%)$ & $27(90 \%)$ & 0.5 \\
\hline U-shaped connection & $0(0 \%)$ & $30(100 \%)$ & $0(0 \%)$ & $30(100 \%)$ & --- \\
\hline Thickness, PostCG<PreCG & $2(6.7 \%)$ & $28(93.3 \%)$ & $2(6.7 \%)$ & $28(93.3 \%)$ & 1 \\
\hline \multicolumn{6}{|l|}{ Left Side } \\
\hline References for the parietal region & $35(13 \%)$ & $235(87 \%)$ & $49(18.1 \%)$ & $221(81.9 \%)$ & $<0.001$ \\
\hline Postcentral gyrus & $0(0 \%)$ & $30(100 \%)$ & $0(0 \%)$ & $30(100 \%)$ & --- \\
\hline Postcentral sulcus & $0(0 \%)$ & $30(100 \%)$ & $0(0 \%)$ & $30(100 \%)$ & --- \\
\hline Posterior ascending ramus/SMG & $8(26.7 \%)$ & $22(73.3 \%)$ & $8(26.7 \%)$ & $22(73.3 \%)$ & 1 \\
\hline Superior temporal sulcus & $8(26.7 \%)$ & $22(73.3 \%)$ & $16(53.3 \%)$ & $14(46.7 \%)$ & 0.021 \\
\hline Angular gyrus & $14(46.7 \%)$ & $16(53.3 \%)$ & $19(63.3 \%)$ & $11(36.7 \%)$ & 0.062 \\
\hline Intraparietal sulcus & $0(0 \%)$ & $30(100 \%)$ & $0(0 \%)$ & $30(100 \%)$ & --- \\
\hline Superior parietal lobe & $2(6.7 \%)$ & $28(93.3 \%)$ & $3(10 \%)$ & $27(90 \%)$ & 1 \\
\hline U-shaped connection & $0(0 \%)$ & $30(100 \%)$ & $0(0 \%)$ & $30(100 \%)$ & --- \\
\hline Thickness, PostCG<PreCG & $3(10 \%)$ & $27(90 \%)$ & $3(10 \%)$ & $27(90 \%)$ & 1 \\
\hline
\end{tabular}
$p<0.05$

Table 5: Performance comparison between T1 GRE and T1 IR GRE.

incidence of three or four interruptions was $16 \%$ on the right and $40 \%$ on the left [16]. Increase in the number of segments hindered the correct identification of this element. Therefore, a method such as T1 IR GRE, which provides higher contrast between the gyri and sulci, was expected to be more precise in recognising the references [10].
Therefore, the findings from this study were consistent with the anatomical data. As for the MRI studies, studies by Naidich et al. 1995 , 1996, 1997 on this region did not mention any percentages for observation in relation to these references $[12,17,18]$. The angular gyrus AG on the right side was the second element to present differences 
between the two techniques, and was better viewed on the right using T1 IR GRE. In the description of the method used to locate the references, the angular gyrus was defined as a continuation of the distal portion of the STS, which can be single or bifid. Analysis of these assertions in relation to anatomic studies by Ono et al. demonstrated that the AG was continuous in relation to the STS in $84 \%$ of cases on the right, and $92 \%$ of cases on the left. When the reference is the continuity of the superior segment of the STS with a bifid aspect, results showed $16 \%$ on the right and $4 \%$ on the left. A new delimitation of the area corresponding to the AG was proposed by Seghier [19]: the AG may be a continuation of either the superior temporal gyrus or the middle temporal gyrus, and extends to the inferior parietal lobule with its middle border defined by the intraparietal sulcus. Its anterior border with the supramarginal gyrus was identified as the descending portion of Jensen's intermediary sulcus [20], while its posterior border was defined by the dorsal part of the anterior occipital sulcus [21]. Assessment of performance between T1 GRE and T1 IR GRE showed a significant difference in the parietal region as a whole both on the right and left sides. The T1 IR GRE technique was superior on both sides. Classical descriptions in the literature in relation to the parietal lobe and current definitions of the parietal lobe can both be found in the studies by Ono et al. [2], Ebeling et al. [22], and Duvernoy [23]. However, these classical anatomical and topographical descriptions contain conflicting points when compared to the current literature. This is a result of three aspects: variability of the anatomic references, inconsistency among authors when naming the elements comprising this region, and divergence when naming same structure. As for the variability of the sulci that limit the parietal lobe PL, only the lateral fissure, the postcentral sulcus, and the intraparietal sulcus are primary. The other sulci are either secondary or tertiary and, as such, are not consistent in terms of appearance. The most constant elements in the parietal lobe are the postcentral sulcus, which establishes its anterior border, and the intraparietal sulcus, which divides the portion located behind the postcentral sulcus into the superior SPL and inferior parietal lobules IPL. The ascending portion of the posterior horizontal ramus PHR on the lateral fissure helps in locating the supramarginal gyrus, which was found around this reference [24]. Inconsistencies in the literature were found in the nomenclature for the sulci that delineate the parietal-temporal-occipital region, most notably in relation to the inferior parietal lobule IPL. Some atlases do not discuss its location at all $[25,26]$, while others such as the ones by Ono et al. [27] and Duvernoy [28] attempt to detail this information. However, those atlases have contradicting reports and differ with regard to the nomenclature of some of the same references. In the study by Ono et al., the two caudal rami of the superior temporal sulcus CRSTS were referred to using three different terms: angular sulcus, anterior occipital sulcus, and an uncommon configuration comprised of two parallel rami. Likewise, Duvernoy called both CRSTS by three different names: ascending posterior segment, horizontal posterior segment, and lateral occipital sulcus. It is important to highlight the fact that although each used three different names, both authors considered the CRSTS to have only two segments. These divergences were demonstrated by comparing what Duvernoy called the ascending posterior segment to what Ono et al called the angular sulcus and the anterior occipital sulcus, or what Duvernoy called the horizontal posterior segment to what Ono et al. called the inferior temporal sulcus and the anterior occipital sulcus [29]. In this context, a new assessment of the references comprising this region is deemed necessary. In a recent re-examination of the sulci comprising the inferior parietal lobule, the existence of three distinct sulci was confirmed. They are related to the STS and ascend toward the IPL. The first ascending arm of the CRSTS was located immediately behind the ascending portion of the PHR on the lateral fissure. The second one was the central arm, located between the two rami and extending dorsally to the intraparietal sulcus IPS. The third arm was in a posterior position in the parietal-occipital junction, and could be shaped like a small sulcus interconnecting horizontally with the central arm or be an elongation of the ventral portion of the anterior occipital sulcus, which was named the temporal-occipital connection sulcus. It is functionally associated with visuospatial process mechanisms connected most notably with locomotion. There are two sulci which are correlated to the IPS and add to the sulci mentioned above, located in the IPL. They are the anterior intermediate parietal sulcus of Jensen, and the posterior intermediate parietal sulcus. The former is usually located in the posterior area of the ascending arm, while the latter is usually located in the posterior area of the middle arm [30]. In this context, there is an attempt to establish a standard for the elements comprising the parietal lobe in response to the lack of consensus as to which the most frequent morphology is, causing the location of the references to depend on the method used to define them and on the characteristics of the studied samples.

\section{References}

1. Mesulam MM (1990) Large-scale neurocognitive networks and distributed processing for attention language and memory. Annals of neurology 28: 597-613.

2. Ono M, Kubik S, Abernathey CD (1990) Parietal and Occipital Lobes In Thieme (ed.) Atlas of the Cerebral Sulci Stutgart: Thieme Medical Publishers, pp: $62-74$

3. Duvernoy HM (1999) Sagital sections In: Springer-Verlag (ed.) The Human Brain - Surface Three-Dimensional Sectional Anatomy with MRI and Blood Supply. New York: Springer-Verlag, pp: 252-309.

4. Grossman CB (1996) Physical principles of computed tomography and magnetic resonance Imaging In: Wilkins $W$ (ed.) Magnetic resonance imaging and computed tomography of head and spine Baltimore Maryland USA: Williams and Wilkins, pp: 10-54

5. Tamraz JC, Comair YG (2006) Brain Cortical Mantle and White Matter Core In: Springer-Verilag editor Atlas of Regional. Anatomy of the Brain Using MRI - With Functional Correlations Heidelberg AM: Springer-Verilag, pp: 51-116.

6. Foundas AL, Weisberg A, Browning CA, Weinberger DR (2001) Morphology of the frontal operculum: a volumetric magnetic resonance imaging study of the pars triangularis. Journal of Neuroimaging 11: 153-159.

7. Keller SS, Highley JR, Garcia-Finana M, Sluming V, Rezaie R, et al. (2007) Sulcal variability stereological measurement and asymmetry of Broca's area on MR images. Journal of Anatomy 211: 534-555.

8. Calabrese M, De Stefano N, Atzori M, Bernardi V, Mattisi I, et al. (2007) Detection of cortical inflammatory lesions by double inversion recovery magnetic resonance imaging in patients with multiple sclerosis. Archives of Neurology 64: 1416-1422.

9. Geurts J, Roosendaal S, Calabrese M, Ciccarelli O, Agosta F, et al. (2011) Consensus recommendations for MS cortical lesion scoring using double inversion recovery. MRI Neurology 76: 418-424.

10. Meyer JR, Roychowdhury S, Russell EJ, Callahan C, Gitelman D, et al. (1996) Location of the central sulcus via cortical thickness of the precentral and postcentral gyri on MR. American journal of neuroradiology, 17: 1699-1706.

11. Achten E, Boon P, De Poorter J, Calliauw L, Van De Kerckhove, et al. (1995) An MR protocol for presurgical evaluation of patients with complex partial seizures of temporal lobe origin. American Journal of Neuroradiology 16: 1201-1213.

12. Naidich TP, Valavanis AG, Kubik S, Taber Kh, Yasargil MG, et al. (1997) Anatomic relationships along the low-middle convexity: Part II: Lesion Localization. International Journal of Neuroradiology 3: 393-409.

13. Wagner M, Jurcoane A, Hattingen E (2013) The U sign: tenth landmark to the central region on brain surface reformatted MR imaging. American Journal of Neuroradiology 34: 323-326.

14. Sim J, Wright CC (2005) The kappa statistic in reliability studies: use interpretation and sample size requirements Physical Therapy 85: 257-268. 
Citation: Sergio Georgeto MD (2017) Morphological Aspects of the Parietal Lobe in the Lateral Surface of the Brain: An in vivo MRI Study. Med Rep Case Stud 2: 130. doi: 10.4172/2572-5130.1000130

Page 7 of 7

15. Byrt T, Bishop J, Carlin JB (1993) Bias prevalence and kappa. Journal of Clinical Epidemiology 46: 423-429.

16. Ono M, Kubik S, Abernathey CD (1990) Temporal Lobe: Lateral Surface In: Thieme editor Atlas of the Cerebral Sulci Stutgart: Thieme Medical Publishers, pp: 78-79.

17. Naidich TP, Valavanis AG, Kubik S (1995) Anatomic relationships along the low-middle convexity: Part I-normal specimens and magnetic resonance imaging. Neurosurgery 36: 517-532.

18. Naidich TP, Brightbill TC (1996) Systems for localizing fronto-parietal gyri and sulci on axial CT and MRI International. Journal of Neuroradiology, 2: 313-338.

19. Seghier ML (2013) The angular gyrus multiple functions and multiple subdivisions, The Neuroscientist Journal 19: 43-61.

20. Ribas GC (2010) The cerebral sulci and gyri Neurosurgical focus, Vol-28.

21. Rademacher J, Galaburda A, Kennedy D, Filipek P, Caviness V, et al. (1992) Human cerebral cortex: localization parcellation and morphometry with magnetic resonance imaging, Cognitive Neuroscience Journal 4: 352-374.

22. Ebeling U, Steinmetz H, Huang Y, Kahn T (1989) Topography and identification of the inferior precentral sulcus in MR imaging. American Journal of Neuroradiology 10: 937-942.

23. Duvernoy HM (1999) Sagital sections In: Springer-Verlag (ed.) The Human Brain - Surface Three-Dimensional Sectional Anatomy with MRI and Blood Supply. New York: Springer-Verlag, pp: 279-280.
24. Caspers S, Aumunts K, Zilles K (2012) Posterior Parietal Cortex: Multimodal Association Cortex In: Mai JK Paxinos G editors. The Human Nervous System London: Elsevier, pp: 1036-1040.

25. Haine D (2008) Neuroanatomy: An Atlas of Structures sections and Systems In: Haine D editor External Morphology of the Central Nervous System Seventh ed Philadelphia PA: Woltters Kluwer Health Lippincott Williams and Wilkins, pp: 10-39.

26. Mancall E, Brock D (2010) Cerebral Hemispheres In: Mancall E Brock D (eds.) Gray's Clinical Neuroanatomy: The Anatomic Basis for Clinical Neuroscience. Philadelphia: Elsevier Saunders, pp: 279-300.

27. Ono M, Kubik S, Abernathey CD (1990) Nomenclature In: Thieme (ed.) Atlas of the Cerebral Sulci Stutgart: Thieme Medical Publishers, pp: 10-13.

28. Duvernoy HM (1999) Surface Anatomy In: Springer-Verlag (ed.) The Human Brain - Surface Three-Dimensional Sectional Anatomy with MRI and Blood Supply. New York: Springer-Verlag, pp: 8-11.

29. Segal E, Petrides M (2012) The morphology and variability of the caudal rami of the superior temporal sulcus European. Journal of Neuroscience 36: 20352053.

30. Petrides M (2012) Sulci and Gyri of Cerebral Cortex In: Petrides M editor The Human Cerebral Cortex An MRI Atlas of Sulci and Gyri in Stereotaxi Space London: Elsevier, pp: 25-53. 